\title{
Abstracts
}

\section{Taming the Expellee Threat in Post-I945 Europe: Lessons from the Two Germanies and Finland}

This article analyses the process through which the dangers posed by millions of forced migrants were defused in continental Europe after the Second World War. Drawing on three countries West Germany, East Germany and Finland - it argues that broad, transnational factors - the cold war, economic growth and accompanying social changes - were crucial in the process. But it also contends that bloc-level and national decisions, particularly those concerning the level of autonomous organisational activity and the degree and type of political and administrative inclusion allowed for the refugees, affected the integration process in significant ways and helped to produce divergent national outcomes.

\section{Everyday Mussolinism: Friends, Family, Locality and Violence in Fascist Italy}

The article uses a range of archival material to commence an investigation into how and with what assumptions Italians lived under a Mussolinian dictatorship which called itself totalitarian. It suggests that 'revolutionary' Fascism - what Emilio Gentile and others have seen as a new 'political religion' - met with some scepticism in the daily behaviour of many Italians. The family, the locality, the most helpful functioning of patron-client networks, the search for special advantage for oneself and 'friends' conditioned Fascist militancy and framed 'ordinary life', all the more because the practice of the dictatorship was itself so shot through with contradictions.

\section{Uncomfortable Questions: Cyprus, October I973-August 1974}

Scholarly research to date has analysed the Cyprus issue from the perspective of Greek-Turkish relations, suggesting that the United States was attempting to strike a balance between them in order to safeguard the cohesion of NATO's southern flank during the cold war. This article, without undermining the validity of previous historical findings on the issue, nevertheless constitutes an attempt to move towards a differing research agenda: it locates Cyprus in the Middle Eastern theatre and suggests that the Yom Kippur war of October I973 may have more linkages to the Cyprus crisis of summer I974 than one may at first sight discern.

\section{Submerging or Clinging On Again? József Antall, Father and Son, in Hungary after 1956}

The aim of this case study of the two Antalls, father and son (the latter became the first Hungarian prime minister after the free elections in 1990) is to present and analyse the period that coincided with the post-I956 development of the Kádár system. Its apparent 
success, efficiency and partial, surrogate, legitimacy has often been explained by the so-called 'compromise' of the Kádárist leadership with Hungarian society after 1956, particularly the 'old intelligentsia' or 'old middle classes'. In fact, while there was an obvious continuity in institutions and ideology between the classic Stalinist regime and that of Kádár, the societal and political practice of the system gradually changed. The Antalls were representative of the inter-war upper middle class (the father) and the participants in the 1956 revolution (the son). Discrimination according to their social background, prevalent in the early I950s, diminished at the turn of the I960s, so that someone descended from the former Christian middle class, like the younger József Antall, could be recruited into the intelligentsia.

\section{War and Political Violence in Twentieth-Century Europe}

This article takes the obvious link between war and political violence in twentieth-century Europe to ask three questions. Did the cause of such a massive upsurge in violence have roots extending beyond the technologies of modern warfare? What shapes the relative propensity of states and societies towards violence? And what is specifically 'modern' (other than the technology of destruction) about mass killing in the twentieth century? It finds answers in the use of popular sovereignty to justify unprecedented ethnic conflict, in a mix of ingredients linked to political culture and contested state legitimacy, and in the role of bureaucracy and technology in the orchestration of large-scale and state-sponsored violence.

\section{Extraits}

\section{Domestiquer les expulsés: leçons de l'Europe de l'après I945}

Cet article analyse les processus par lesquels furent désamorcés les problèmes posés par les millions de personnes déplacées en Europe après la Deuxième Guerre Mondiale. A travers l'exemple de trois pays (les deux Allemagne et la Finlande), on y rappelle que les facteurs macro politiques et sociaux furent cruciaux dans cette évolution (la Guerre Froide, la croissance économique et les changement sociaux qu'elle induisit). Mais on y suggère aussi que les décisions nationales et régionales (au niveau des blocs), en particulier celles qui touchaient à la liberté d'association, d'organisation et d'action d'une part, et aux procédures d'intégration administrative et politique de l'autre, jouèrent un rôle non négligeable, identifiable notamment dans la divergence des voies nationales ici examinées.

\section{Le fascisme au jour le jour: amis, famille, localité et violence dans l'Italie mussolinienne}

Cet article puise à toute une gamme de sources pour inaugurer une enquête sur les modalités et les catégories de perception du fascisme par les italiens qui ont vécu sous ce régime qui s'autoproclama totalitaire. Le fascisme 'révolutionnaire', celui que Emilio Gentile et d'autres ont parfois considéré comme une 'nouvelle religion', ne semble pas avoir été épousé avec effusion par les les italiens dans leur vie de tout les jours. La famille, la localité, le fonctionnement des réseaux clientélistes, la recherche d'avantages pour soi et pour les 'amis' conditionnèrent davantage l'existence ordinaire et le militantisme fasciste lui-même. Et ce d'autant plus que l'exercice même de la dictature était rempli de contradictions.

\section{Des questions embarassantes: Chypre octobre I973-août 1974}

Jusqu'à présent, on a surtout envisagé la question chypriote sous l'angle des relations grécoturques, en suggérant que les Etats-Unis tentèrent d'y tenir la balance égale entre ces deux pays pour maintenir intact le flanc sud de l'OTAN dans une période de guerre froide. Cet article, sans remettre en cause la validité de ces approches, suggère néanmoins le besoin 
d'une contextualistion plus large. Il replace l'affaire de Chypre au milieu des affaires moyenorientales, et suggère que la guerre du Kippour pourrait avoir plus de liens avec la crise de Chypre qu'il n'y parait.

\section{Au creux et à la crête de la vague: Jozsef Antall père et fils dans la Hongrie kadarienne}

Cette étude de cas se propose d'explorer la période de développement du système Kàdàr après I956, à travers les trajectoires des Antall pére et fils (ce dernier fut le premier ministre de la Hongrie après les élections libres de I990). L'apparent succès de ce système fut souvent attribué à la mise en place d'un compromis entre les fidèles de Kàdàr et la société hongroise, en particulier avec les groupes de la bourgeoisie traditionnelle, la vieille intelligentsia. Et en vérité, s'il y eût une vraie continuité institutionnelle et idéologique entre la version stalinienne du régime communiste et sa forme 'kadarisée', la pratique sociale et politique du régime changea progressivement. Les Antall incarnent respectivement la haute bourgeoisie de l'entre deux guerres (pour le père) et les insurgés de 1956 (le fils). La discrimination en fonction des origines sociales, très nette dans les années I950 envers le père, s'atténua assez fortement au début des années 1960 pour qu'un descendant de la haute bourgeoisie chrétienne, en l'occurrence Joszef Antall fils, puisse être intégré à l'intelligentsia du régime.

\section{Guerre et violence politique dans l'Europe du 2oème siècle}

Cet article part du lien évident entre guerre et violence politique dans l'Europe contemporaine pour poser trois questions. La cause de cette élan massif de violence a-t-elle des raisons qui dépassent les simples modifications des technologies de la guerre? Qu'est ce qui informe la propension des états et des sociétés à la violence? Et qu'y-a-t-il de spécifiquement 'moderne' en dehors des techniques de destruction - dans les massacres de masse du 2oème siècle? Les réponses à ces questions sont recherchées dans les usages de la souveraineté populaire pour justifier le conflit ethnique, dans les traits mêlés de la culture politique et de la contestation de la légitimité de l'état, et dans le rôle conjoint de la bureaucratie et de la technologie pour orchestrer une violence de grande échelle patronnée par l'appareil d'état.

\section{Kurzfassungen}

\section{Wie das Flüchtlingsproblem im Nachkriegseuropa eingedämmt wurde: Lehren aus den beiden deutschen Staaten und Finnland}

Der Artikel analysiert wie die von Millionen von Flüchtlingen ausgehende Bedrohung nach dem Zweiten Weltkrieg in Kontinentaleuropa entschärft wurde. Am Beispiel von Westdeutschland, Ostdeutschland und Finnland wird argumentiert, dass weitreichende transnationale Faktoren wie der Kalte Krieg, das Wirtschaftswachstum und daraus folgende soziale Veränderungen in diesem Prozess entscheidend waren. Desweiteren wird die These vertreten, dass Entscheidungen auf Block- und nationaler Ebene, insbesondere diejenigen, die das Ausmass unabhängig organisierter Aktivität und den Grad und die Art politischer und administrativer Einbeziehung für die Flüchtlinge betrafen, den Integrationsprozess in signifikanter Weise beeinflusst haben und so zu unterschiedlichen nationalen Resultaten führten.

\section{Alltäglicher Mussolinismus: Freunde, Familie, lokale Gemeinschaft und Gewalt im faschistischen Italien}

Basierend auf umfangreichem Archivmaterial, widmet sich der Artikel der Erforschung der Frage, wie und unter welchen Annahmen die Italiener unter Mussolinis Diktatur, die sich 
selbst als totaliär bezeichnete, lebten. Er weist darauf hin, dass der 'revolutionäre Faschismus', den Emilio Gentile und andere als neue 'politische Religion' sahen, im täglichen Leben vieler Italiener auf Skepsis stieß. Familie, lokale Gemeinschaft und das hilfreiche Funktionieren von Patron-Klienten Verhältnissen bestimmten die faschistische Militanz mit und bildeten den Rahmen für das tägliche Leben ein, umso mehr als die Praxis der Diktatur an sich mit Widersprüchen durchsetzt war.

\section{Unangenehme Fragen: Zypern, Oktober I973-August 1974}

Die Wissenschaft hat das Thema Zypern bis jetzt aus der Perspektive der griechisch-türkischen Beziehungen betrachten, unter der Annahme, dass die USA versuchten, zwischen beiden Staaten ein Gleichgewicht herzustellen, um so die NATO-Südflanke im Kalten Krieg zu stabilisieren. Ohne die Gültigkeit früherer historischer Untersichungen zum Thema zu widerlegen, versucht der Artikel dennoch zu einer neuen Forschungsperspektive zu gelangen: er plaziert das Thema in den Rahmen des Nahostkonflikts und deutet an, dass der Yom Kippur Krieg vom Oktober 1973 enger mit der Zypernkrise im Sommer I974 verknüpft war, als auf den ersten Blick erkennbar wird.

\section{Untertauchen oder sich wieder anpassen? Joszef Antall, Vater und Sohn, im Ungarn nach 1946}

Das Ziel dieser Studie des Falls der beiden Antalls, Vater und Sohn (der später zum ersten Premierminister Ungarns nach den freien Wahlen I990 wurde) ist es, den Zeitraum während der Entwicklung des Kádár-Systems nach 1956 zu beschreiben und zu analysieren. Sein offenbarer Erfolg, seine Effizienz und die partielle Ersatzlegitimität ist oft mit dem sogenannten 'Kompromiss' der Kádáristischen Führung mit der ungarischen Gesellschaft nach I 956 erklärt worden, im speziellen mit der 'alten Intelligentsia' oder der 'alten Mittelschicht'. Tatsächlich veränderte sich trotz der offensichtlichen Kontinuität in Institutionen und Ideologie zwischen dem klassichen Stalinistischen Regime und demjenigen Kádárs schrittweise die gesellschaftliche und politische Praxis des Systems. Die Antalls repräsentierten die obere Mittelklasse der Zwischenkriegszeit (Vater) und die Teilnehmer am Aufstand von I956 (Sohn). Die in den frühen fünziger Jahren herrschende Diskrimierung aufgrund ihres sozialen Hintergrunds verminderte sich zu Beginn der I960er Jahre, so dass eine der früheren christlichen Mittelschicht enstammende Person, wie Joszef Antall junior, für die Intelligentsia gewonnen werden konnte.

\section{Krieg und politische Gewalt im Europa des 20. Jahrhunderts}

Dieser Artikel verwendet die offensichtliche Verbindung zwischen Krieg und politischer Gewalt im 20. Jahrhundert in Europa, um drei Fragen zu stellen. Wurzelte die massive Ausweitung von Gewalt in Faktoren jenseits der Technologie moderner Kriegfuihrung? Was formt die relative Neigung von Staaten und Gesellschaften zu Gewalt? Und was ist spezifisch 'modern' an den Massentötungen des 20. Jahrhunderts (abgesehen von der Destruktivität der Technologie)? Er findet Antworten im Gebrauch der Volkssouveränität, welche noch nie dagewesene ethnische Konflikte rechtfertigte, in einer Mischung von Elementen politische Kultur und hinterfragter Legitimität des Staates, sowie in der Rolle von Bürokratie und Technologie in der Orchestrierung von staatlich unterstützter Gewalt im großen Ausmaß. 